\title{
Gerenciamento de interfaces para prover comunicação heterogênea em redes compostas por múltiplos UAVs
}

\author{
Laura Michaella B. Ribeiro ${ }^{* \ddagger}$, Ivan Muller ${ }^{\dagger}$ e Leandro Buss Becker* \\ ${ }^{*}$ Prog. de PG em Engenharia de Automação e Sistemas (PPGEAS), UFSC - Florianópolis, SC, Brasil \\ Email:1.michaella@posgrad.ufsc.br, ivan.muller@ufrgs.br, leandro.becker@ufsc.br \\ ${ }^{\dagger}$ Departamento de Engenharia Elétrica (DELET), UFRGS - Rio Grande do Sul, RS, Brasil \\ $\ddagger$ Instituto Federal do Amazonas, Campus Manaus Distrito-Industrial, Manaus, AM, Brasil
}

\begin{abstract}
Manter a conectividade em voo entre múltiplos UAVs é um grande desafio técnico. Porém, muitas vezes, isso se torna indispensável para o sucesso das tarefas e alcance dos objetivos de uma missão. Nesse contexto, visando ampliar a confiabilidade na entrega de mensagens, o presente artigo propõe um sistema gerenciador de interfaces capaz de prover uma comunicação heterogênea em redes multi-UAVs. Esse sistema é constituído por uma árvore de decisão que, a partir de parâmetros sensoriados dinamicamente pelo meio (vazão da rede, RSSI e SNR), escolhe qual interface de comunicação emprega uma maior confiabilidade nas conexões disponíveis. Cenários de simulação foram gerados utilizando as ferramentas NS-3 e SUMO, empregando o gerenciador com as interfaces IEEE 802.11n 2.4 GHz e 802.11p 5 GHz. A performance do gerenciador é avaliada em termos de vazão de rede, quantidade de pacotes enviados/recebidos, RSSI, intensidade de ruído recebido e a relação sinal-ruído. Em comparação ao desempenho obtido pelas interfaces aplicadas de forma homogênea, o gerenciador de interfaces proposto apresentou uma menor flutuação de desempenho na vazão, mesmo variando as classes de acesso das mensagens a serem enviadas (AC_BE, AC_BK, AC_VI, AC_VO). Isso implicou em maiores taxas de vazão da rede, maior quantidade de pacotes recebidos, menor quantidade de pacotes perdidos e uma melhor relação sinal-ruído, contribuindo para obtenção de conexões mais estáveis.
\end{abstract}

Index Terms-Redes sem-fio, UAVs, Redes ad-hoc, Mobilidade, interfaces de comunicação.

\section{INTRODUÇÃO}

Maximizar a entrega de pacotes e melhorar a qualidade das transmissões são requisitos dinamicamente necessários para constituir redes sem fio compostas por nós de alta mobilidade [1], [2]. De modo a atender essa necessidade, algumas pilhas de protocolos apresentam em sua camada de acesso ao meio, alterações nos esquemas de associação dos nós, como a redução no intervalo de geração de quadros CTS, RTS e ACK e a diminuição do tamanho do cabeçalho dos pacotes, reduzindo assim, o tempo de resposta na entrega das mensagens.

Um exemplo disso é a pilha de protocolos IEEE 802.11p aplicada na comunicação entre veículos, que possui a alternativa de permitir o envio de mensagens dentro de uma faixa de alcance, baseada no geoposicionamento dos nós sem a necessidade de autenticação à rede [3], [4]. Outras alternativas para manter a confiabilidade destas redes incluem mecanismos complementares a um protocolo de comunicação, que atuam gerenciando as atribuições dos canais de frequência, sistemas de algoritmos eficientes que otimizam a qualidade na troca de mensagens e esquemas de reposicionamento dos nós, de modo a melhorar a sua intensidade da conexão [5]-[7].

Manter conexões sem fio estáveis entre nós móveis de alta mobilidade, como os nós constituídos por UAVs (Veículos Aéreos Não Tripulados) trata-se de um campo de pesquisa com vários desafios, devido a caracterítica de mobilidade, flexibilidade e topologias variantes que constituem estas redes conhecidas como FANETs (do inglês, Fly Ad-Hoc Networks) [8]. Algumas dessas características incluem em boa parte dos casos, tempo de voo limitado (como em desastres naturais), resistência à interferências (por exemplo, sobreposição de canais) e limites impostos pela tecnologia sobre a extensão da cobertura de sinal [9].

$\mathrm{O}$ uso de diferentes interfaces ou protocolos de comunicação (redes heterogêneas) pode ser uma alternativa viável na melhoria da capacidade de sobrevivência e confiabilidade de redes compostas por múltiplos UAVs. Tipicamente, cada nó (UAV) envia diferentes tipos de mensagens, tais como: voz, video, dados dos sensores, dados dos atuadores e sinais de coordenação de tarefas. Deste modo, o uso das diferentes características intrínsecas presentes em diferentes padrões de comunicação, podem proporcionar maior adaptabilidade da rede, melhorando a estabilidade das conexões.

Dependendo da aplicação é preciso atender diferentes requisistos de rede, como largura de banda, potência, e vazão de rede necessários para a efetiva comunicação [10], [11]. Em virtude disso, a tarefa de aplicar um único padrão de comunicação para suportar diferentes tráfegos de rede, se torna mais crítica, podendo comprometer a integridade da missão.

Neste contexto, soluções que empregam redes heterogêneas podem ser úteis para o problema de manter a conectividade entre esses tipos de nós, buscando qualidade e confiabilidade na entrega das mensagens [12].

Baseando-se nisto, este artigo propõe um gerenciador de interfaces para prover comunicação heterogênea em redes compostas por múltiplos UAVs. O objeto principal do geren- 
ciador é manter a conexão com QoS (Quality of Service) entre os nós móveis para o envio de diferentes tipos de serviço entre eles durante toda a missão (em tempo de voo).

Neste artigo, para avaliar se uma conexão possui ou não QoS, são utilizados alguns parâmetros de rede como condições de avaliação para integrar uma heurística de decisão composta por um sistema de pontos. Estas condições envolvem a quantidade de bytes recebidos, a quantidade de bytes perdidos, a vazão da rede (throughput), a potencia de sinal recebido RSSI e a relação sinal-ruído (SNR - Signal to Noise Ratio).

O gerenciador de interfaces toma decisões a partir de uma árvore de decisão, cujos níveis são constituídos pelas condições de avaliação dinâmica da rede, a partir da soma de pontos obtidos para cada interface de comunição empregada. Cada ponto é somado a partir dos avanços dos níveis em direção à escolha de uma interface. Assim, o gerenciador escolhe a interface com uma maior soma de pontos, ou seja, aquela que possui melhor performance nas condições de avaliação empregadas. Como as condições de avaliação são parâmetros comuns nas redes de comunicação sem fio e o gerenciador atua acima da camada de enlace de rede é esperado a possibilidade de extensões futuras para um número maior de interfaces. $\mathrm{O}$ presente artigo avalia a proposta gerenciando apenas duas interfaces: IEEE 802.11n $2.4 \mathrm{GHz}$ e IEEE 802.11 p $5 \mathrm{GHz}$. Estas foram escolhidas por permitir adicionar à FANET diferentes características de frequência, largura de banda, alcance e composição dos pacotes.

O restante do artigo está organizado como segue, algumas outras soluções são apresentadas para prover comunicação entre múltiplos UAVs na Seção II. A Seção III, descreve o gerenciador e o algoritmo de decisão, a Seção IV descreve os cenários de experimentação aplicados, a Seção V detalha os experimentos e resutados obtidos e, por fim, a Seção VI descreve as conclusões obtidas e os direcionamentos para os trabalhos futuros.

\section{TRABALHOS RELACIONADOS}

A definição de um padrão de comunicação para redes compostas por UAVs deve levar em consideração a alta mobilidade dos nós, a qual implica diretamente na estabilidade dos enlaces de comunicacação. Com isso, alguns trabalhos concentram esforços para definir estes padrões de acordo com a aplicação da rede, utilizando um único padrão de comunição (sistemas homogêneos) ou mais de um padrão (sistemas heterogêneos), buscando garantias de conectividade entre os nós ao longo da missão [9].

Dentre os trabalhos que aplicam soluções homogêneas pode-se citar [13], onde os autores utilizaram uma arquitetura de comunicação em pilha dupla aplicando uma comunicação bidirecional a um nó UAV, o qual estabelece comunicação com a rede de sensores sem fio (RSSF) e um nó sink utilizando o mesmo rádio. Além de estabelecer o enlace, esta arquitetura possibilita atender outras diferentes características de QoS, tais como best-effort e a confiabilidade responsáveis pela manutenção dos enlaces UAV-RSSF e UAV-Sink, respectivamente. Os resultados demonstraram que é possível manter uma comunicação bidirecional usando um único rádio, a partir de uma infraestrutura de comunicação flexível, que define a pilha de protocolo a ser aplicada de acordo com o comunicação detectada, se MDC (mobile data collector) ou se comunicações com o sink.

Em [14] é proposto um sistema multi-UAVs para prover comunicação em tempo real. O principal objetivo dos autores é demonstrar que com hardware de baixo custo é possível constituir FANETs que atendam aplicações de tempo real. Os autores empregaram o Ar.Drone 2.0, uma unidade de voo comercial de arquitetura fechada, juntamente com uma placa Raspberry Pi Model B com duas interfaces IEEE 802.11n, uma para permitir a comunicação com o outro UAV e outra para receber o streaming de video do voo. No entanto, os autores realizaram apenas testes de latência utilizando dois UAVs, um estático e outro que realiza uma tragetória fixa ao longo do alcance de sinal do UAV estático, sem avaliar os efeitos do uso de duas interfaces de protocolos iguais nessa comunicação o que pode acarretar em interferências devido a coexistência de redes.

O trabalho em [15] investiga o problema do uso de UAVs para redistrinuição de tráfego em áreas de desastres naturais, baseado nas demandas dos usuários de uma determinada área, definido por um modelo neural reverso que adota padrões de demanda e funções de densidade para enviar UAVs para áreas específicas. Redes LTE 5G foram utilizadas para prover maior alcance e maior capacidade de usuários conectados.

Em [16] é apresentado uma solução empregando mudanças na camada MAC do padrão de comunicação IEEE 802.11p, utilizando mecanismos de otimização que visam evitar a ineficiência na priorização do tráfego (tempo ocioso), resultante do uso do mecanismo de acesso EDCA (enhanced distributed channel access). O EDCA é aplicado pelo padrão IEEE 802.11p para priorização de mensagens em classes de acesso (AC) (voz, video, best effort e background). O objetivo é propor uma solução para o problema da confiabilidade no tráfego de mensagens de segurança em redes veiculares. Esta solução também pode ser considerada por redes compostas por UAVs, pois considera características de mobilidade também existentes nestas redes. $\mathrm{O}$ mecanismo proposto permitiu uma redução de até $20 \%$ na taxa de perda de pacotes melhorando o throughput da rede a cada adição de novos veículos, apresentando desempenhos parecidos com o IEEE 802.11p EDCA sem alterações para os casos com quantidades pequenas de veículos.

Dentre as soluções heterogêneas, em [17] é apresentado uma arquitetura de comunicação em que os nós UAV se comunicam por uma rede celular e uma rede IEEE 802.11p. O objetivo da proposta é prover um algoritmo de posicionamento seguro para UAVs em enxames, usando quadros beacon de RSSI enviados pelos UAVs como estimativa de distância. Os autores consideram um cenario de comunicação, onde os UAVs usam a rede celular $(3 \mathrm{G} / 4 \mathrm{G})$ para o envio de mensagens para estação base e interfaces IEEE 802.11p para mensagens U2U, operando na frequência de $5.9 \mathrm{GHz}$. A gerência de rede é centralizada, pois apenas mensagens beacon são trocadas 
entre os UAVs. Os resultados demonstraram que as taxas de incertezas em relação ao calculo das distâncias entre UAVs, geradas por RSSI recebidos pela triangulação de nos vizinhos é reduzida a uma distância de até $50 \mathrm{~m}$, entre eles, porem aumenta gradativamente acima desta distância.

Um dos grandes desafios remanescentes é deployment de redes multi-UAVs em áreas remotas, usando o alcance de transmissões sem fio que operam em bandas não licenciadas. Em [18] é apresentado o uso de algoritmos de retransmissão e roteamento desenvolvidos em sistemas ROS (Robot Operating System), para prover um maior alcance em transmissões sem fio em redes multi-UAVs. O sistema utiliza um padrão que opera na frequência de $2.4 \mathrm{GHz}$ para comunicção entre o UAV líder do enxame e estação base e $5 \mathrm{GHz}$ em transmissões para U2U. O gerenciamento é centralizado, pois os UAVs avançam suas posições de acordo com a melhoria do sinal RSSI recebido entre o lider do enxame e a estação base. Os resultados demonstraram se tratar de uma solução escalavel, extendendo o alcance da comunicação a cada nova inserção de UAVs, melhorando a conectividade da rede como um todo.

Em [19] é apresentado um sistema de comunicação dividindo os UAVs em cluster-heads e membros do cluster. Os UAVs cluster-head são equipados por interfaces de tecnologias LTE e WiFi em modo AP (Access Point) e os membros do cluster são equipados unicamente com interfaces WiFi em modo estação. Os cluter-heads podem conectar a um servidor de rede privada virtual VPN (Virtual Private Network) para receber comandos de controle por meio da interface LTE e recebem dados dos sensores por meio das interfaces $\mathrm{WiFi}$ presentes nos membros do cluster. Os autores avaliaram o sistema usando consumo de banda, latência e RSS (Received Strength Signal). Os resultados demonstraram que a latência é estável até uma distância de $18 \mathrm{~m}$ entre os cluster-heads e os cluster membros com suporte para tranmissões de video em tempo real, apresentando taxas entre 4-6 Mbps e RSS acima de $-60 \mathrm{dBm}$ considerando interfaces de comunicação integradas preexistentes nos UAVs (sem extensões de hardware).

Os trabalhos apresentados, em geral, não avaliam as condições de rede em termos de estabilidade das conexões considerando as diferentes classes de serviço a serem enviadas pela rede para atender determinada aplicação. No caso dos trabalhos que aplicam comunicações heterogêneas em suas soluções, uma grande parte adota condições pré-existentes de missão, acerca de qual interface será utilizada para um determinado fim reduzindo a adaptabilidade da rede a eventos inesperados.

Visando apresentar uma proposta para estas possibilidades, maximizar a entrega de pacotes e a vazão de rede, minimizando os impactos dos ruídos provenientes do meio, este artigo propõe um gerenciador de interfaces para prover uma comunicação heterogênea em redes compostas por múltiplos UAVs. A conexão é avaliada periodicamente de acordo com um intervalo de tempo predefinido para uma nova execução do gerenciador $N_{1}, N_{2}$. A cada intervalo de amostras, a rede é avaliada considerando diferentes condições/parâmetros previamente configurados.

\section{GERENCIAMENTO DE INTERFACES}

Esta seção apresenta uma proposta para gerenciamento de interfaces, o qual utiliza parâmetros de redes típicos para a tomada de decisões. Os parâmetros são capturados por sensoriamento de espectro através das camadas MAC e PHY. Desta forma, o gerenciador proposto pode ser visto como um módulo que permite a adição de mais interfaces, podendo serem distintas daquelas aplicadas nos experimentos deste artigo.

Vale ressaltar que cada padrão de comunicação aplicado por uma interface implica em largura de banda, frequência de sinal e tipos de associações inerentes a este. Tais características são assumidas de forma independente ao funcionamento do gerenciador.

O principal objetivo do gerenciador proposto é aumentar a confiabilidade na entrega de mensagens entre nós UAVs pertencentes à uma rede sem fio ad-hoc. Para isto, o gerenciador avalia se uma conexão estabelecida pelas interfaces aplicadas possui ou não QoS, considerando os diferentes tipos de classes de acesso (do inglês, access class (AC)) das mensagens a serem enviadas: voz (VO), video (VI) e dados (BE e BK).

Para analisar a qualidade de serviço (QoS) de uma conexão estabelecida entre os nós vizinhos e, consequentemente a confiabilidade do sinal, o gerenciador avalia algumas condições (parâmetros) sensoriados do meio, as quais compõe os níveis de uma árvore de decisão composta por uma heurística de soma de pontos. Estas condições são compostas por: quantidade de bytes recebidos, quantidade de bytes perdidos, vazão da rede (throughput), potência de sinal recebido (RSSI) e relação sinal-ruído (SNR).

Desta forma, ao considerar uma comunicação heterogênea, um conjunto INT é formado pelas interfaces de comunicação que compõe o sistema, onde as condições de decisão devem ser avaliadas para cada interface de comunicação $I N T_{x}$, baseada nos últimos pacotes recebidos.

A heurística proposta baseia-se em um sistema de ponto, onde cada interface acumula pontos a partir do seu desempenho ao longo da missão, de forma que o gerenciador soma um toda vez que uma condição de decisão $c^{i}$ for mais satisfatória para uma determinada interface $I N T_{x}$, conforme formalizado na equação 1 ,

$$
I N T_{x}^{c^{i}}=\left\{\begin{array}{c}
1, \mathrm{se} \mathrm{c}_{x}^{i} \text { for o melhor desempenho } \forall x \in I N T \\
0, \text { caso contrário }
\end{array}\right.
$$

Desta forma, o gerenciador de interfaces atua de forma embarcada em um conjunto $X=\left\{x_{k}\right\}_{k=1}^{|X|}$ de UAVs que compõe uma rede ad-hoc estabelecida por uma missão. Cada UAV representa um nó móvel $x$ pertencente ao conjunto $|X|$, o qual deve ser capaz de se comunicar com os outros nós, de modo a possibilitar o envio do status de execução das tarefas (sinalização), dados de voz e video, permitindo a execução de objetivos compartilhados em tempo de missão. 
Uma árvore de decisão é empregada para avaliar qual interface de comunicação propiciará maior confiabilidade na conexão para o envio de mensagens. A qualidade de uma comunicação é avaliada a partir das conexões estabelecidas de um nó e seus vizinhos, tomando como referência o enlace entre os nós vizinhos dentro da área de cobertura das interfaces usadas, uma vez que a distância entre eles variam com o tempo.

Assim, a confiabilidade de uma conexão é definida pela soma dos pontos obtidos a partir dos avanços através dos níveis da árvore em direção ao estado alvo de decisão, comparando as performances das interfaces, em cada condição.

Esta proposta apresenta como estudo de caso o envio de mensagens entre UAVs no contexto de missões coordenadas e colaborativas de busca e regaste (SAR-Search and Rescue), ou seja, missões em que os nós precisam trocar mensagens entre eles durante toda a missão e, em curtos intervalos de tempo, considerando uma transmissão de sinal em espaço livre de obstáculos e uma quantidade fixa de nós.

Para melhorar a confiabilidade das conexões em uma rede ad-hoc, cada nó necessita estar conectado com ao menos um nó vizinho por meio de um enlace eficiente, ou seja, com qualidade de link e vazão de rede suficientes para estabelecer uma conexão confiável com qualidade de serviço.

A Tabela I descreve as métricas utilizadas como critério de decisão pelo gerenciador de interface. Cada uma das métricas é aplicada em forma de uma condição $c^{i}$ na equação 2 .

TABELA I

CONDIÇÕES UTILIZADAS COMO REQUISITOS PARA TOMADA DE DECISÕES

\begin{tabular}{ll}
\hline Condição $(c)$ & Métricas de Rede \\
\hline $\mathrm{c}_{1}$ & Vazão de Rede \\
\hline $\mathrm{c}_{2}$ & Total de Bytes Enviados (carga útil) \\
\hline $\mathrm{c}_{3}$ & Total de Bytes Recebido (carga útil) \\
\hline $\mathrm{c}_{4}$ & RSSI (Received Strength Sinal Indicator) \\
\hline $\mathrm{c}_{5}$ & SNR (Signal to Noise Ratio) \\
\hline
\end{tabular}

Desta forma, o gerenciador de interfaces captura através de sensoriamento do meio ou calcula cada condição de decisão, mediante os últimos quadros recebidos pelos UAVs, verificados a cada intervalo de amostras $N=n_{0}, n_{1}, n_{2}, \ldots, n_{n}$ definidos para uma nova captura de parâmetros. Assim, o valor de uma condição $c^{i}$ utilizado na heurística de decisão, trata-se da média dos valores obtidos nas últimas amostras sensoriadas/calculadas $\sum_{n-1}^{n} c_{n}^{i}$ para cada interface pertencente ao conjunto $I N T$.

Quando uma interface não for a escolhida pelo gerenciador para o envio de mensagens, a mesma permanece emitindo beacons, permitindo uma avaliação contínua das condições de decisão, de acordo com a variação dinâmica do meio,

$$
I N T_{x}^{c^{i}}=\frac{\sum_{n-1}^{n} c_{n}^{i}}{(n+1)}, \forall n \in N
$$

Cada condição da Tabela I descreve um nível na altura da árvore de decisão da Figura 1, o qual só atinge o estado alvo após a verificação do desempenho de todas as condições

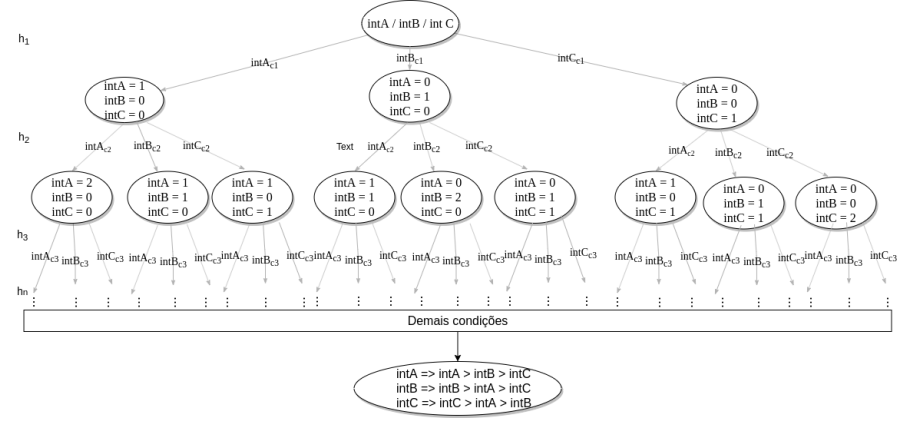

Fig. 1. Árvore de decisão utilizada pelo gerenciador de interfaces

definidas pelas interfaces que compõem o conjunto $I N T$. Para evitar que uma interface apresente o valor de uma determinada condição sempre nulo, para os casos em que gerenciador não realize nenhuma decisão por esta interface, ou no caso de inicio da missão, são consideradas pelo menos dois intervalos de amostras de transmissão por cada interface, por exemplo, para três interfaces utilizadas, as seis amostras de teste iniciais correspondem a troca de mensagens de teste entre os UAVs por meio de cada uma das três interfaces alternadamente não implicando em interfaces escolhidas pelo gerenciador.Por esse motivo, as amostras iniciais entre 0-10s não são consideradas na plotagem dos gráficos provenientes dos experimentos.

Desta forma, a árvore de decisão é estabelecida, a partir de uma heurística de acúmulo de pontos para cada interface $I N T_{x}$, sendo a decisão de cada nível pela interface de melhor desempenho na condição que compõe o nível. $\mathrm{O}$ estado alvo consistirá da interface com melhor desempenho em um maior número de condições caracterizando uma conexão mais confiável.

A árvore de decisão aplicada é classificada como não perfeita, pois apenas uma única interface é escolhida para transmissão das mensagens, gerando um estado alvo proveniente de uma subárvore não completa por cada intervalo de amostra de tempo $n$.

A equação 3 apresenta a heurística composta pelos pontos somados por cada interface $\sum_{h}^{H} I N T_{x}^{h}$ ao longo dos níveis $h$ do conjunto de níveis que compõem uma árvore $H$.

$$
\begin{array}{r}
\mathrm{INT}_{x}=\sum_{h}^{H} I N T_{x}^{h}, \forall h \epsilon H \\
\mathrm{INT}_{x}>I N T, \log x_{k}=I N T_{x}
\end{array}
$$

Assim, a interface com o maior número de pontos $\max \left(I N T_{x}\right)$ será a escolhida $\left(x_{k}=I N T_{x}\right)$ por um UAV $\left(x_{k}\right)$ para o próximo envio de mensagens, a partir do intervalo $n$ de amostras definido para uma nova verificação. As demais interfaces não escolhidas, ficam em estado de escuta para receber as mensagens enviadas por outros UAVs que as estejam utilizando e enviando quadros beacon para atualizar as amostras das condições de decisão. 


\section{DESCRIÇÃO DOS CENÁRIOS DE EXPERIMENTAÇÃO}

Os resultados foram gerados a partir de dois cenários de experimentação um contendo 3 nós móveis UAV e outro contendo 10 nós. Cada cenário é composto por linhas retas que definem as distâncias a serem percorridas por cada UAV, constituídas por waypoints, que são pontos formados por coordenadas que conectados entre si definem a trajetória de cada veículo, a partir de um ponto inicial (waypoint 1). Neste caso, o ponto inicial $(0 \mathrm{x}, 0 \mathrm{y})$ constitui o ponto de origem e destino comum a todas as trajetórias, variando em torno de até $1.8 \mathrm{~m}$ (cenário 2) para posicionamento inicial e final dos UAVs, permitindo que iniciem suas missões ao mesmo tempo, evitando colisões na partida/chegada. Cada veículo possui também diferentes velocidades máximas a serem empregadas ao longo da trajetória com uma acelaração de $0.8 \mathrm{~m} / \mathrm{s}^{2}$, desaceleração $\left(4.5 \mathrm{~m} / \mathrm{s}^{2}\right)$ e um imperfeição de $0.5 \%$ na execução da trajetória do veículo. Os diferentes waypoints e velocidades máximas de cada veículo são apresentados nas legendas das Figuras 2 e 3.

Utiliza-se o SUMO (Simulator of Urban Mobility) na versão 1.0.1 para definir os waypoints e as características de mobilidade de cada nó (aceleração, velocidade), os quais foram definidos utilizando as configurações de um UAV de pequeno porte. O primeiro cenário é representado na Figura 2.

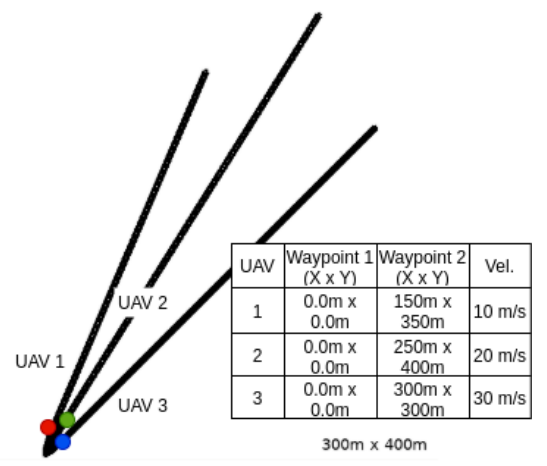

Fig. 2. Primeiro cenário de experimentos compostos por 3 nós móveis UAV.

Este cenário é definido em um área de $300 \mathrm{~m} \mathrm{x} 400 \mathrm{~m}$ na qual são definidas 3 trajetórias para 3 nós UAVs que se movimentam em velocidades máximas distintas: nó UAV 1 $(10 \mathrm{~m} / \mathrm{s})$,nó UAV $2(20 \mathrm{~m} / \mathrm{s})$ e nó UAV $3(30 \mathrm{~m} / \mathrm{s})$.

A Figura 3 apresenta o segundo cenário de experimentação, composto por uma área total de $600 \mathrm{~m}$ x $800 \mathrm{~m}$ sendo percorrida por 10 nós UAVs, que se dividem da seguinte

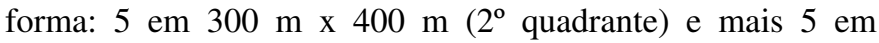
outros $300 \mathrm{~m}$ x $400 \mathrm{~m}$ em sentido contrário ( $3^{\circ}$ quadrante) em relação a um ponto de origem comum de partida/chegada. O objetivo deste cenário é representar um número maior de UAVs percorrendo uma área também maior, permitindo que os nós alcancem maiores distâncias em relação uns aos outros. Buscase o limite da área de cobertura das interfaces de comunicação utilizadas (IEEE 802.11n $2.4 \mathrm{GHz}$ e IEEE 802.11p $5 \mathrm{GHz}$ ), visando avaliar o desempenho do gerenciador de interfaces e das interfaces empregadas de forma separada, em situações em que o nós alcançam o limite de cobertura, sem utilizar gateways, repetidores ou RSUs (roadside units).

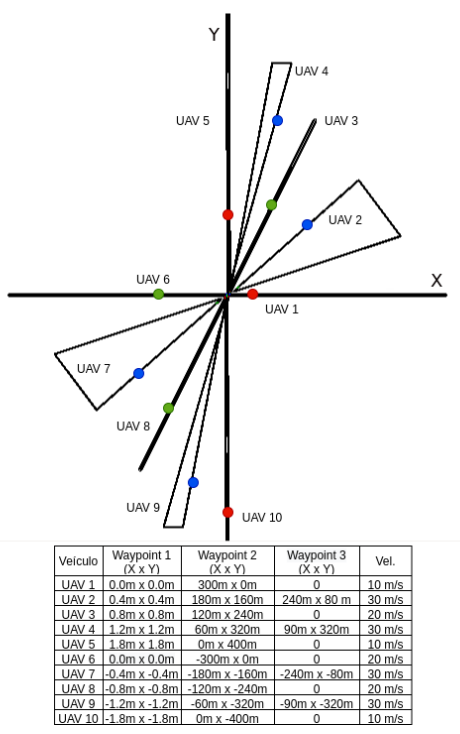

Fig. 3. Segundo cenário de experimentos compostos por 10 nós móveis UAV.

Neste cenário, os UAVs que apresentam até três waypoints aplicam uma velocidade máxima de $30 \mathrm{~m} / \mathrm{s}$, os que apresentam até dois waypoints com o segundo contendo uma das coordenadas iguais a 0 , aplicam uma velocidade máxima de $20 \mathrm{~m} / \mathrm{s} \mathrm{e}$, os demais, apresentam velocidade máxima de $10 \mathrm{~m} / \mathrm{s}$. O intuito dessa variação na quantidade de waypoints e velocidades empregadas por cada veículo é simular diferentes distâncias a serem percorridas por diferentes tipos de UAVs, padronizando as distâncias maiores a serem percorridas para UAVs mais rápidos, conforme visto na Figura 3. A Tabela II apresenta os demais parâmetros utilizados por ambos os cenários de experimentação.

TABELA II

CONFIGURAÇão dos CENÁRIOS

\begin{tabular}{|c|c|}
\hline Parâmetro & Configuração \\
\hline Tipo de Rede & Redes ad-hoc \\
\hline Modelo de Atenuação & Espaço livre de obstáculos \\
\hline Potência de Tx/Rx & $20 \mathrm{dBm}(200 \mathrm{~mW})$ \\
\hline Velocidade média dos nós & $10 \mathrm{~m} / \mathrm{s}, 20 \mathrm{~m} / \mathrm{s}$ and $30 \mathrm{~m} / \mathrm{s}$ \\
\hline Modelo de Desvanescimento & Nakagami \\
\hline Tempo de Simulação & 90 segundos (um percurso completo) \\
\hline Intervalo de Amostras & $100 \mathrm{~ms}$ \\
\hline Tamanho do Pacote & 1472 bytes \\
\hline Interface A & IEEE $80211 \mathrm{n} 2.4 \mathrm{GHz}$ \\
\hline Área de Cobertura Int. A & $300 \mathrm{~m}$ \\
\hline Frequência do Canal Int. A & $2.432 \mathrm{GHz}(\mathrm{CH} \mathrm{5-6)}$ \\
\hline Interface B & IEEE $80211 \mathrm{p} \mathrm{5} \mathrm{GHz}$ \\
\hline Área de Cobertura Int. B & $350 \mathrm{~m}$ \\
\hline Frequência do Canal Int. B & $5.860 \mathrm{GHz}(\mathrm{CH} \mathrm{172)}$ \\
\hline
\end{tabular}

O modelo Friis de propagação em espaço livre é usado na simulação como modelo de atenuação de sinal. No entanto, para incluir efeitos de desvanecimento de sinal, um modelo estatístico de sombreamento Nakagami é aplicado, uma vez que pode ocorrer dos nós estarem fora da linha de visão (LOS 
- Line-Of-Sight), ou até mesmo estarem na LOS, mas em distancias maiores que o alcance das suas antenas

O modelo Nakagami é empregado usando uma distribuição Rayleigh, considerando as variações na força do sinal devido ao desvanecimento multipercurso. $\mathrm{O}$ canal de frequência adotado pela Interface A (IEEE $802.11 \mathrm{n} 2.4 \mathrm{GHz}$ ) é composto pela soma dos canais canais $5(2.432 \mathrm{GHz})$ e $6(2.437 \mathrm{GHz})$ de $20 \mathrm{MHz}$ cada, sobre a banda ISM (Industrial Scientific and Medical) de $2.4 \mathrm{GHz}$, possibilitando uma área de cobertura de $300 \mathrm{~m}$. A Interface B é composta pelo padrão IEEE 802.11p $5 \mathrm{GHz}$ utilizando o canal 172 na frequência de $5.860 \mathrm{GHz}$ de $10 \mathrm{MHz}$ de largura de banda sobre o espectro de frequências $5.9 \mathrm{GHz}(5.850-5.925 \mathrm{GHz})$ da banda U-NII (Unlicensed National Information Infrastructure), com área de cobertura de até $350 \mathrm{~m}$.

\section{EXPERIMENTOS E RESULTADOS}

Os experimentos foram conduzidos aplicando-se as interfaces utilizadas pelo gerenciador de forma homogênea em experimentos separados (IEEE 802.11n 2.4 GHz e IEEE 802.11p $5 \mathrm{GHz})$ e de forma heterogênea utilizando o Gerenciador de Interfaces. O tempo de cada experimento é de $100 \mathrm{~s}$ compreendendo uma execução completa da missão (90 s), mais o descarte das primeiras amostras coletadas, de modo a minimizar os efeitos de execução inicial das redes na validação dos resultados.

As amostras são coletadas em intervalos de $100 \mathrm{~ms}$ e dado o volume de dados coletados cerca de 1000 amostras para cada conjunto de experimentos, as mesmas foram agregadas em grupos de $10 \mathrm{~s}$ dos quais os pontos das curvas correspondem a média obtida nesses grupos. O intervalo de confiança adotado é de $95 \%$, definido pela variação obtida nas amostras.

A Figura 4 apresenta a vazão média da rede obtida por 3 nós móveis UAV que correspondem ao cenário 1 de experimentos. Nesta é possível visualizar que nas amostras iniciais de $10 \mathrm{~s}$ a $40 \mathrm{~s}$ o desempenho dos três experimentos é o mesmo, com uma variação entre as amostras $40 \mathrm{~s}$ e $70 \mathrm{~s}$, onde o experimento relacionado ao gerenciador de interface apresenta um desempenho de vazão superior em $1 \mathrm{MB} / \mathrm{s}$ (megabits por segundo) representando um volume maior de dados recebido pelos nós UAV neste intervalo.

A vazão média da rede se apresenta muito próxima a zero nos três experimentos nas amostras iniciais, em função da referência adotada ser em megabits por segundo $(\mathrm{MB} / \mathrm{s})$, correspondentes aos tráfegos constituídos por mensagens de sinalização e quadros beacon (AC_BE (best effort) e AC_BK (background)). A referência $\mathrm{MB} / \mathrm{s}$ foi adotada devido a vazão da rede alcançar valores em torno 1 a $3 \mathrm{MB} / \mathrm{s}$ a partir do envio de mensagens de video e voz nos experimentos aplicando o Gerenciador de Interfaces e a interface IEEE 802.11 n $2.4 \mathrm{GHz}$ de forma homogênea.

Vale ressaltar, que a cada intervalo de $10 \mathrm{~s}$ o tipo de classe de acesso de serviço das mensagens enviadas é alterado, dentre os 4 tipos de serviço AC_BE, AC_BK, AC_VI (video) e $\mathrm{AC} \_\mathrm{VO}$ (voz), sendo as primeiras amostras de 0-10 s constituídas por AC_BE e, assim sucessivamente, onde o intervalo

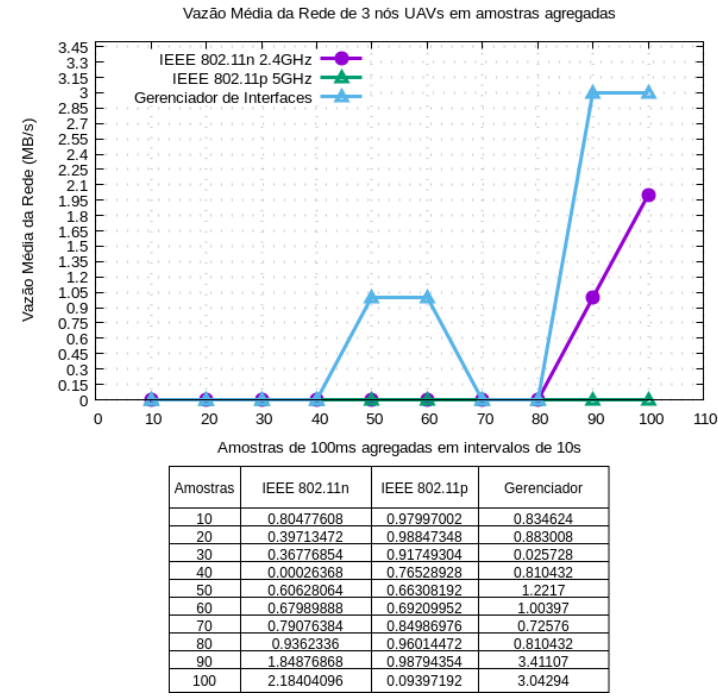

Fig. 4. Cenário 1 - Vazão média da rede por amostras agregadas de 10s.

de 40 s a 80 s refere-se a segunda sequência de envio das diferentes classes de serviço. No início da terceira sequncia de envio (em $80 \mathrm{~s}$ ) percebe-se um aumento na vazão de dados de cerca de $2 \mathrm{MB} / \mathrm{s}$ na curva referente ao IEEE 802.11n $2.4 \mathrm{GHz}$ e na curva referente ao Gerenciador de Interfaces, este aumento refere-se as distâncias mais curtas entre os nós em seu retorno para o waypoint de partida, aumentando a potência de sinal na recepção. A curva referente a interface IEEE 802.11p não apresentou maiores variações na vazão ao longo do experimento, mantendo valores muito próximo de vazão, mesmo no início de uma nova sequencia de envio. Isto se deve, principalmente a largura de banda desta tecnologia.

A Figura 5 apresenta a vazão obtida pela rede no cenário 2. Percebe-se uma vazão maior obtida nos três experimentos em função do maior número de UAVs (10 nós) e, consequentemente, um número maior de dados trafegados. Do mesmo modo, são aplicados sucessivos envios a cada $10 \mathrm{~s}$ de diferentes tipos de classes de acesso de serviço, onde entre as amostras $40 \mathrm{~s}$ e $80 \mathrm{~s}$ observou-se uma variação no aumento da vazão neste intervalo (segunda sequência de envio). Na curva referente à interface IEEE $802.11 \mathrm{n} 2.4 \mathrm{GHz}$ foi possível atingir picos de $7 \mathrm{MB} / \mathrm{s}$ durante o envio de pacotes pertencentes a classe $\mathrm{AC}_{-} \mathrm{VI}$ e de $5 \mathrm{MB} / \mathrm{s}$ no envio de pacotes da classe de serviço $\mathrm{AC}_{-} \mathrm{VO}$.

A curva referente a interface IEEE 802.11p $5 \mathrm{GHz}$ apresentou uma diminuição na vazão da rede durante o inicio da segunda sequencia de envio das classes referente a AC_BE em $40 \mathrm{~s}$ e AC_BK em $50 \mathrm{~s}$, iniciando um aumento de cerca de $1 \mathrm{MB} / \mathrm{s}$ no envio de pacotes do tipo AC_VI em $60 \mathrm{~s}$. O baixo volume de dados refere-se a largura de banda de $10 \mathrm{MHz}$ desta interface, onde a partir da terceira sequencia de envio das classes em $80 \mathrm{~s}$ apresenta taxas de vazão de $3.25 \mathrm{MB} / \mathrm{s}$.

A terceira curva diz respeito ao desempenho do gerenciador de interfaces, onde é possível notar um comportamento com menor flutuação de desempenho na vazão, entre $3 \mathrm{MB} / \mathrm{s}$ e 


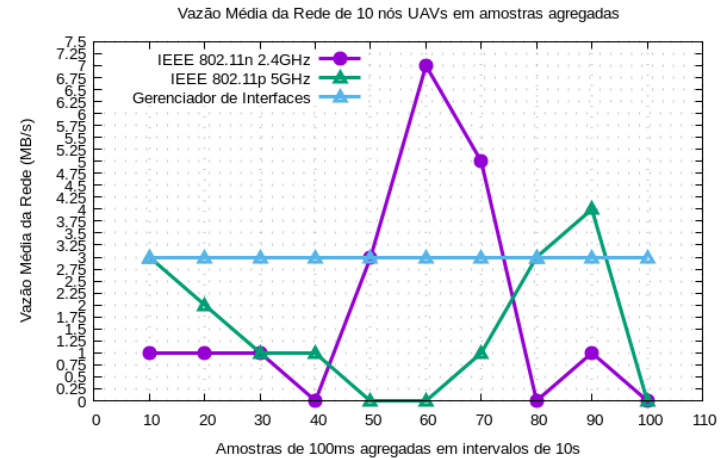

Fig. 5. Cenário 2 - Vazão média da rede por amostras agregadas de $10 \mathrm{~s}$.

3.25 MB/s. Observa-se que as diferentes classes de serviço não implicam em maiores variações na vazão, permitindo uma maior estabilidade na comunicação com maior vazão de dados.

Destaca-se também que os valores obtidos de vazão dizem respeito à média obtida pelo desempenho da rede, que no caso do experimento gerenciador de interfaces manteve-se em torno dos $3 \mathrm{MB} / \mathrm{s}$ descrevendo um comportamento aparentemente fixo em virtude da alta quantidade de amostras coletadas para um número maior de UAVs e o chaveamento aplicado pelo gerenciador.

As curvas flutuantes apresentam as interfaces quando aplicadas de forma homogênea. Elas caracterizam um comportamento típico de redes sem fio compostas por nós de alta mobilidade, no qual dependendo da interface usada e das condições de enlace entre os nós, implica em conexões altamente instáveis. Isso reforça a complexidade do problema, i.e., de manter conexões confiáveis neste tipo de rede.

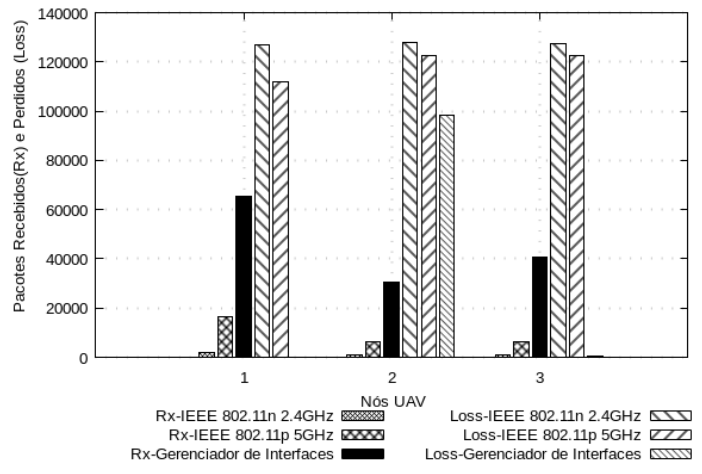

Fig. 6. Cenário 1 - Quantidade de pacotes recebidos (Rx) e pacotes perdidos (Loss) por UAV.

A Figura 6 apresenta a quantidade de pacotes recebidos e perdidos por cada nó UAV para o cenário 1 . Foram enviados 128.651 pacotes por cada nó considerando todos os tipos de classes de acesso. É possível visualizar na Figura 6 que o gerenciador de interfaces empregou a melhor proporção de pacotes recebidos/perdidos do que as demais interfaces empregadas de forma homogênea. O nó UAV 1 recebeu cerca de 60.000 pacotes, o nó UAV 230.000 pacotes e o nó UAV 3
40.000 pacotes, sem a existência de pacotes perdidos. Assim, o gerenciador de interfaces apresentou melhor taxa de entrega de pacotes do que as interfaces empregadas de forma homogênea, com um maior volume de pacotes enviados e entregues.

Comportamento semelhante pode ser verificado na Figura 7, onde com exceção dos nós UAV 2 e 3 , o gerenciador apresentou a maior quantidade de pacotes recebidos e, em todos os nós, a menor quantidade de pacotes perdidos. Neste cenário, o volume total de pacotes enviados para cada nó foi de 38.595. Neste cenário, dentre as interfaces homogêneas, o melhor rendimento foi da interface IEEE 802.11n.

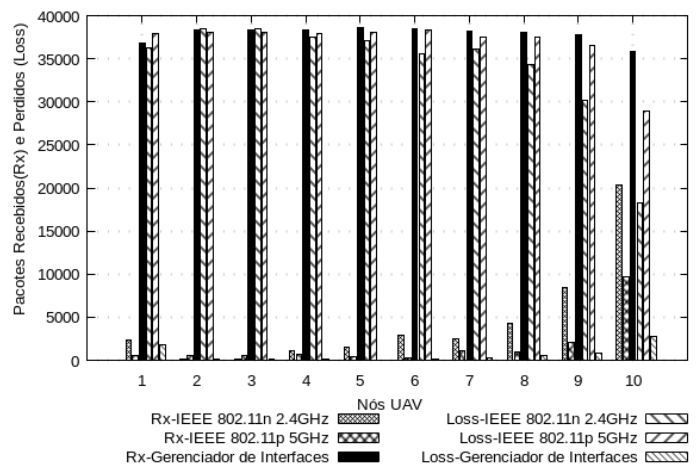

Fig. 7. Cenário 2 - Quantidade de pacotes recebidos $(\mathrm{Rx})$ e pacotes perdidos (Loss) por UAV.

A Tabela III apresenta a média dos parâmetros sensoriados pelos nós a partir de 5 amostras aleatórias de ambos os cenários coletadas durante a execução dos experimentos. É possível verificar que o gerenciador de interfaces apresentou valores de SNR superiores a 0.66 nas 5 amostras coletadas, intensidade de sinal recebio (RSSI) em torno de $-68 \mathrm{dBm}$ e potência de ruído recebido menores que $-93 \mathrm{dBM}$. Estas condições promovem transmissões com QoS suficientes para envio e recepção de dados utilizando interfaces 802.11. As interfaces empregadas de forma homogênea também apresentaram RSSI em níveis considerados muito bons ou excelentes para este tipo de comunicação $(-56 \mathrm{dBm}$ até $-68 \mathrm{dBm})$, destacando o menor ruído recebido pela interface IEEE 802.11p, dada a característica de menor susceptibilidade das frequências de $5 \mathrm{GHz}$.

Ao longo do experimento percebeu-se variações nas potências de sinal recebido, geradas durante o chaveamento entre as interfaces, um vez que uma desconexão é realizada para efetivar a troca de interface (nos casos em que o gerenciador decidiu pela troca). No entanto, os nós não tiveram desconexão total, uma vez que a latência gerada para reconexão não impactou na entrega das mensagens, restabelecendo a conexão em um intervalo desprezível de tempo. Como o custo das operações de uma árvore binária é $\log n$ o overhead gerado na execução do algoritmo do gerenciador de interfaces também é desprezível.

Isso demonstra que diferentes interfaces podem ser aplicadas de acordo com as condições dinâmicas do meio, buscando estabelecer conexões mais confiáveis ao ajustar qual 
interface propiciará um melhor desempenho em um determinado instante, a partir das diferentes métricas avaliadas.

\section{CONCLUSÕES E TRABALHOS FUTUROS}

Missões que utilizam sistemas multi-UAVs necessitam de uma maior capacidade de entrega de mensagens e confiabilidade em sua entrega. Nesse contexto, um sistema gerenciador de interfaces heterogêneo foi proposto. $\mathrm{O}$ gerenciador permitiu ajustar a interface de comunicação de acordo com a dinamicidade do meio e as variações de mobilidade dos nós, permitindo uma maior vazão de dados, maior quantidade de pacotes recebidos, menor quantidade de pacotes perdidos com desempenho favorável de relação sinal-ruído para este tipo de comunicação.

O gerenciador também apresentou uma menor flutuação da vazão da rede mesmo com o aumento da quantidade de nós e mediante a variação das diferentes classes de serviço a serem enviadas, mantendo a transmissão em níveis favoráveis. Em comparação às interfaces empregadas de forma homogênea $e$ considerando as métricas de avaliação adotadas, o gerenciador contribuiu para uma maior estabilidade na rede.

Como trabalhos futuros espera-se adicionar outras condições/parâmetros de avaliação à heurística, de modo a considerar também o tipo de mensagem a ser enviada (voz, dados ou video) comparando o seu desempenho com outras heurísticas. Também parece ser importante analisar em mais detalhes as situações em que mais de uma interface é usada em determinado instante.

Outro foco futuro diz respeito à adição de esquemas de retransmissão de sinal à solução, não somente para prover uma maior confiabilidade na entrega de mensagens, mas também uma melhor capacidade de sobrevivência da rede relacionada aos nós que apresentam baixa recepção de sinal durante a missão, estendendo o alcance de sinal à distâncias maiores.

TABELA III

RSSI, RUÍDO RECEBIDO E SNR

\begin{tabular}{|c|c||c|c|}
\hline Interface & RSSI & Ruído Recebido & SNR \\
\hline IEEE 802.11n 2.4 GHz & -64.3673 & -93.9073 & 0.685434 \\
\hline IEEE 802.11n 2.4 GHz & -68.5251 & -93.9178 & 0.729628 \\
\hline IEEE 802.11n 2.4 GHz & -68.5845 & -93.9179 & 0.7302620 \\
\hline IEEE 802.11n 2.4 GHz & -69.2706 & -93.9207 & 0.737543 \\
\hline IEEE 802.11n 2.4 GHz & -68.6618 & -93.9343 & 0.730956 \\
\hline IEEE 802.11p 5 GHz & -56.6779 & -96.7504 & 0.585816 \\
\hline IEEE 802.11p 5 GHz & -61.891 & -96.8081 & 0.639316 \\
\hline IEEE 802.11p 5 GHz & -67.318 & -96.7876 & 0.695523 \\
\hline IEEE 802.11p 5 GHz & -66.4942 & -96.7945 & 0.686963 \\
\hline IEEE 802.11p 5 GHz & -66.3235 & -96.7887 & 0.68524 \\
\hline Gerenciador de Interfaces & -62.7718 & -93.9487 & 0.66815 \\
\hline Gerenciador de Interfaces & -69.4198 & -93.9507 & 0.738895 \\
\hline Gerenciador de Interfaces & -70.1968 & -93.9244 & 0.747376 \\
\hline Gerenciador de Interfaces & -71.9332 & -93.849 & 0.766478 \\
\hline Gerenciador de Interfaces & -72.0667 & -93.8873 & 0.767586 \\
\hline
\end{tabular}

\section{AGRADECIMENTOS}

Este trabalho foi desenvolvido com o apoio do Governo do Estado do Amazonas por meio da Fundação de Amparo à Pesquisa do Estado do Amazonas, com a concessão de bolsa de estudo e, agradecemos ao IFAM e a UFSC pelo suporte recebido.

\section{REFERÊNCIAS}

[1] A. Sayyed, "Exploiting and optimizing mobility in wireless sensor networks." 2016

[2] J. Scherer, B. Rinner, S. Yahyanejad, S. Hayat, E. Yanmaz, T. Andre, A. Khan, V. Vukadinovic, C. Bettstetter, and H. Hellwagner, "An Autonomous Multi-UAV System for Search and Rescue," Proceedings of the First Workshop on Micro Aerial Vehicle Networks, Systems, and Applications for Civilian Use - DroNet '15, pp. 33-38, 2015. [Online]. Available: http://dl.acm.org/citation.cfm?doid=2750675.2750683

[3] D. Jiang and L. Delgrossi, "IEEE 802.11p: Towards an international standard for wireless access in vehicular environments," IEEE Vehicular Technology Conference, pp. 2036-2040, 2008.

[4] IEEE 802.11p, "IEEE Standard for Information technology- Local and metropolitan area networks- Specific requirements-Part 11: Wireless LAN Medium Access Control (MAC) and Physical Layer (PHY) Amendment 6: Wireless Access in Vehicular Environments," IEEE Std 802.11p-2010 (Amend.IEEE Std 802.11-2007 as amended by IEEE Std 802.11k-2008, IEEE Std 802.11r-2008, IEEE Std 802.11y-2008, IEEE Std 802.11n-2009, and IEEE Std 802.11w-2009), pp. 1-51, 2010.

[5] T. Watanabe, J. Takahashi, and Y. Tobe, "Investigation of an algorithm to maximize the information observed by multiple autonomous UAVs," in 2017 IEEE SENSORS, 2017, pp. 1-3.

[6] J. H. Park, S. C. Choi, J. Kim, and K. H. Won, "Unmanned Aerial System Traffic Management with WAVE Protocol for Collision Avoidance," International Conference on Ubiquitous and Future Networks, ICUFN, vol. 2018-July, pp. 8-10, 2018

[7] K. P. Hui, D. Phillips, and A. Kekirigoda, "Beyond line-of-sight range extension in contested environments with OPAL using autonomous unmanned aerial vehicles," 2017 27th International Telecommunication Networks and Applications Conference, ITNAC 2017, vol. 2017-Janua, pp. $1-5,2017$

[8] I. Bekmezci, O. K. Sahingoz, and Temel, "Flying Ad-Hoc Networks (FANETs): A survey," Ad Hoc Networks, vol. 11, no. 3, pp. 1254-1270, 2013.

[9] Y. Saleem, M. H. Rehmani, and S. Zeadally, "Integration of Cognitive Radio Technology with Unmanned Aerial Vehicles," J. Netw. Comput. Appl., vol. 50, no. C, pp. 15-31, 2015. [Online]. Available: http://dx.doi.org/10.1016/j.jnca.2014.12.002

[10] E. Yanmaz, S. Hayat, J. Scherer, and C. Bettstetter, "Experimental performance analysis of two-hop aerial 802.11 networks," in Wireless Communications and Networking Conference (WCNC), 2014 IEEE. IEEE, 2014, pp. 3118-3123.

[11] Menegol, "Multi-Agent Coordination Applied to UAVs." 2018.

[12] B. A. Lima, C. H. O. O. Quev^, R. L. Gomes, and J. C. Jr, "Improving the Communication of Heterogeneous Vehicular Networks through Clusterization," pp. 1-6, 2018

[13] A. Sayyed, G. M. de Araújo, J. P. Bodanese, and L. B. Becker, "Dualstack single-radio communication architecture for UAV acting as a mobile node to collect data in WSNs," Sensors (Switzerland), vol. 15, no. 9, pp. 23 376-23 401, 2015.

[14] I. Bekmezci, I. Sen, and E. Erkalkan, "Flying Ad Hoc Networks ( FANET ) Test Bed Implementation," 2015 7th International Conference on Recent Advances in Space Technologies (RAST), pp. 665-668, 2015.

[15] V. Sharma, M. Bennis, and R. Kumar, "UAV-Assisted Heterogeneous Networks for Capacity Enhancement," IEEE Communications Letters, vol. 20, no. 6, pp. 1207-1210, 2016.

[16] X. Lei and S. H. Rhee, "A new backoff scheme with collision detection for IEEE $802.11 \mathrm{p}$ vehicular networks," IEEE Vehicular Technology Conference, vol. 2017-Septe, pp. 1-5, 2018

[17] R. S. Yokoyama, B. Y. L. Kimura, and E. dos Santos Moreira, "An architecture for secure positioning in a UAV swarm using RSSI-based distance estimation," ACM SIGAPP Applied Computing Review, vol. 14, no. 2, pp. 36-44, 2014. [Online]. Available: http://dl.acm.org/citation.cfm?doid=2656864.2656867

[18] S. P. Yamaguchi and F. Karolonek, "Autonomous Position Control of Multi-Unmanned Aerial Vehicle Network Designed for Long Range Wireless Data Transmission," Proceedings of the 2017 IEEE/SICE International Symposium on System Integration, vol. 7, pp. 5-10, 2017.

[19] B. Y. Chang, Y. C. Chen, and L.-c. Wang, "Design and Implementation of UAV-enabled Flying Access Points,” pp. 3-4, 2018. 